\title{
EEG correlation during the solving of simple and complex logical-mathematical problems
}

\author{
Jahaziel Molina del Río ${ }^{1,2}$ • Miguel Angel Guevara ${ }^{2}$ Marisela Hernández González ${ }^{2}$ Rosa María Hidalgo Aguirre ${ }^{1,2}$. \\ Manuel Alejandro Cruz Aguilar ${ }^{3}$
}

Published online: 21 February 2019

(C) The Psychonomic Society, Inc. 2019

\begin{abstract}
Solving logical-mathematical word problems is a complex task that requires numerous cognitive operations, including comprehension, reasoning, and calculation. These abilities have been associated with activation of the parietal, temporal, and prefrontal cortices. It has been suggested that the reasoning involved in solving logical-mathematical problems requires the coordinated functionality of all these cortical areas. In this study was evaluated the activation and electroencephalographic (EEG) correlation of the prefrontal, temporal, and parietal regions in young men while solving logical-mathematical word problems with two degrees of difficulty: simple and complex. During the solving of complex problems, higher absolute power and EEG correlation of the alpha and fast bands between the left frontal and parietal cortices were observed. A temporal deactivation and functional decoupling of the right parietaltemporal cortices also were obtained. Solving complex problems probably require activation of a left prefrontal-parietal circuit to maintain and manipulate multiple pieces of information. The temporal deactivation and decreased parietal-temporal correlation could be associated to text processing and suppression of the content-dependent reasoning to focus cognitive resources on the mathematical reasoning. Together, these findings support a pivotal role for the left prefrontal and parietal cortices in mathematical reasoning and of the temporal regions in text processing required to understand and solve written mathematical problems.
\end{abstract}

Keywords EEG correlation $\cdot$ Mathematical word problems $\cdot$ Mental calculation $\cdot$ Prefrontal cortex

Solving logical-mathematical word problems is a complex task that requires numerous cognitive operations, including comprehension, reasoning, calculation, knowledge of previously learned mathematical rules, as well as manipulating the new information given by the components of the problem itself. Leron (2004) has proposed that, regardless of the degree of difficulty, solving logical-mathematical problems requires

Miguel Angel Guevara

mguevara@cencar.udg.mx

1 Laboratorio de Neuropsicología, Centro Universitario de los Valles, Universidad de Guadalajara, Carretera Guadalajara-Ameca Km. 45.5, C.P. 46600 Ameca, Jalisco, México

2 Laboratorio de Correlación Electroencefalográfica y Conducta, Instituto de Neurociencias, Universidad de Guadalajara, Francisco de Quevedo, 180. Col. Arcos-Vallarta, C.P. 44130 Guadalajara, Jalisco, México

3 Dirección de Investigaciones en Neurociencias, Laboratorio de Cronobiología y Sueño, Instituto Nacional de Psiquiatría "Ramón de la Fuente Muñiz", Mexico City, México three levels of mathematical thinking. The first level corresponds to rudimentary arithmetic or basic mathematical skills, such as subitizing or addition and subtraction. The second, known as informal mathematics, refers to the use of diagrams, figures, and everyday analogies; while the third, called formal mathematics, is characterized by the use of logical relations or syllogisms - like "if P, then Q" - which have been associated with the act of reasoning (Goel \& Dolan, 2003), one of the pivotal processes required to solve problems of this kind (Leron, 2003; Mahmood, Othman, \& Yusof, 2012). All three levels of mathematical thinking require the participation of cognitive processes that have been studied and consolidated in the triple code model proposed by Dehaene and Cohen (1995). This code consists of three categories of mental representations of numbers - namely, the verbal word frame, the visual Arabic number form, and the analog magnitude representation.

Neuroimaging techniques have revealed the neural bases of the aforementioned categories. The first activation pathway depends on the modality of the stimuli, so that if the problem is presented verbally, activation corresponds to the auditory verbal 
network (Eger, Sterzer, Russ, Giraud, \& Kleinschmidt, 2003), while if the elements of the problem are received through the visual pathway, recognition of the numbers is associated with the visual network, including the inferior and mid occipital cortex. For subitizing and counting (Piazza, Mechelli, Butterworth, \& Price, 2002) with semantic or asemantic associations (Fias, 2001), an area often activated in both cases is the parietal region, which is associated with the magnitude of the quantities involved (Dehaene, Molko, Cohen, \& Wilson, 2004; Eger et al., 2003; Le Clec'H et al., 2000).

One of the main cognitive processes required to solve mathematical problems is working memory (Ashcraft \& Krause, 2007; DeStefano \& LeFevre, 2004), which involves the prefrontal cortex, especially the dorsolateral region (Zago et al., 2001). In fact, it has been reported that subjects with damage to the frontal region show deficits in the manipulation of multiple pieces of information, though their basic arithmetical processes remain intact (Besnard et al., 2014). Similarly, greater activation of the dorsolateral prefrontal cortex has been observed in subjects as they attempt to solve problems that required two operations to obtain the answer, compared with those that required only one (Prabhakaran, Rypma, \& Gabrieli, 2001).

The parietal region, especially the intraparietal sulcus, participates in solving mathematical problems (Knops \& Willmes, 2014). Activation of these areas seems to depend on the characteristics of the problem, since greater activation of the intraparietal sulcus has been reported during solving of two-digit problems (e.g., 15×32) compared with one-digit problems (e.g., $7 \times 3$; Zago et al., 2001). Some studies have associated simple calculation processes with parietal or parieto-occipital regions (Burbaud et al., 1995; Dehaene, Spelke, Pinel, Stanescu, \& Tsivkin, 1999; Levin et al., 1996), as well as algebra processing (Waisman, Leikin, Shaul, \& Leikin, 2014), whereas more complex mathematical tasks that require multiple calculations with intermediate steps seem to require frontal regions as well (Burbaud et al., 1995; Grafman, Passafiume, Faglioni, \& Boiler, 1982; Jackson \& Warrington, 1986). This may be due to an increase in the demands on working memory that are necessary to maintain and manipulate the intermediate products during the problemsolving process.

Another cortical area that participates in information retrieval when people attempt to solve word problems is the temporal cortex (Prabhakaran et al., 2001; Zago et al., 2001). In addition, a pivotal role of this cortical area has been demonstrated in text processing (Fasotti, Eling, \& Bremer, 1992; Fasotti, Eling, \& Houtem, 1994), since previous studies that analyzed the comprehension and reasoning of textual material have shown prominent activations in temporal and frontal regions (Haier \& Camilla, 1995; Just, Carpenter, Keller, Eddy, \& Thulborn, 1996; Nichelli et al., 1995; Partiot, Grafman, Sadato, Flitman, \& Wild, 1996).
Recording electroencephalographic activity (EEG) is a noninvasive procedure with high temporal resolution that allows researchers to obtain information on brain functioning during different behavioral and cognitive states. The data obtained from EEG recordings are grouped in bands by frequency. The most commonly used bands are delta $(\delta, 1.5-3.5 \mathrm{~Hz})$, theta $(\theta, 3.5-7.5 \mathrm{~Hz})$, alpha1 $(\alpha 1,7.5-10.5 \mathrm{~Hz})$, alpha2 $(\alpha 2$, $10.5-13.5 \mathrm{~Hz})$, betal $(\beta 1,13.5-19.5 \mathrm{~Hz})$, beta2 $(\beta 2,19.5-30$ $\mathrm{Hz})$, and gamma $(\gamma, 31-50 \mathrm{~Hz})$. The low frequencies in frontal regions (e.g., theta and delta) have been related to facilitating working memory tasks (Carrillo-de la Peña \& GarcíaLarrea, 2007) and identifying the elements of cognitive tasks (Cunillera et al., 2012). In posterior regions, in contrast, low frequencies have been associated with the performance of visual spatial tasks during continuous periods (Jap, Lal, \& Fischer, 2010). Changes in the alpha range have been related to attentional processes and the inhibition of irrelevant information (Cooper, Croft, Dominey, Burgess, \& Gruzelier, 2003; Foxe \& Snyder, 2011; Payne, Guillory, \& Sekuler, 2013; Sauseng, Klimesch, Schabus, \& Doppelmayr, 2005), while the fast bands (beta1, beta2, gamma) appear to be associated with interneuronal communication of inhibitory networks (Whittington, Traub, Kopell, Ermentrout, \& Buhl, 2000) and information transfer between regions (Engel \& Fries, 2010).

The interaction that occurs among different brain areas to perform cognitive processes is determined by their anatomical and functional connections (Mesulam, 1994). The former consists of bundles of axons that form fasciculi and connect different brain regions, such as the frontal and parietal areas, through the superior longitudinal fasciculus, and the temporal pole with frontal regions through the uncinated fasciculus (Gerig, Gouttard, \& Corouge, 2004; Mori et al., 2002). Functional connections - or synchronization - among brain areas has been studied by electroencephalographic correlation (rEEG), which can provide an index of short-range and longrange functional relations between brain areas (Guevara and Corsi-Cabrera 1996; Harris \& Gordon, 2015; Shaw, 1984; Shaw, O'Connor, \& Ongley, 1977; Thatcher, Biver, \& North, 2007; Varela, Lachaux, Rodriguez, \& Martinerie, 2001).

Electroencephalographic studies conducted while subjects are solving mathematical problems have revealed participation by the left central, temporal, parietal, and right frontal regions (Wang, Chen, Zhao, \& Zou, 2010) and the posterior parietal area (Rousell, Catherwood, Edgar, \& Design, 2012). Also, there are reports that activation of, and synchronization between, frontal and parieto-occipital areas in the theta and alpha bands increase during working memory tasks and in relation to the degree of difficulty of the problem (Dimitriadis, Sun, Thakor, \& Bezerianos, 2016; Sauseng et al., 2004; Zarjam, Epps, Lovell, \& Fang Chen, 2012). Subhani, Malik, Kamil, and Saad (2016) observed a characteristic EEG coherence profile during the solving of mathematical problems that included a stress condition. Meanwhile, 
using depth electrodes in human intracranial recordings, Halgren, Boujon, Clarke, Wang, and Chauvel (2002) observed phase synchrony in the alpha band in occipital, parietal, frontal, and Rolandic regions during periods of mental calculation and working memory maintenance. Additionally, the magnetoencephalographic recordings of human cortical ongoing activity made by Palva, Palva, and Kaila (2005) showed that mental calculation is associated with enhanced frontoparietal alpha band phase synchrony.

Although activation of several cortical areas has been associated with the demands of the mathematical tasks presented, we do not know how the degree of EEG synchronization between the prefrontal and posterior cortices changes during the reasoning or syllogism applied to solve logicalmathematical word problems. Thus, the aim of this study was to characterize the activation (absolute power) and degree of cortical EEG correlation in young men while they solved simple and complex logical-mathematical word problems. Considering the pivotal role that the prefrontal and parietal regions play in manipulating multiple pieces of information, mental calculation, and working memory, we hypothesized that higher activation and enhanced synchronization of the alpha and fast bands between these cortical areas would be found while subjects attempted to solve complex mathematical problems. Similarly, in light of the participation of temporal regions in information retrieval and text processing, we expected to detect changes in the alpha and fast bands as participants sought to solve such complex problems. Finally, better performance was predicted on simple problems, because it is assumed that complex problems require higher cognitive demands.

\section{Material and method}

\section{Participants}

Eighteen young men with a mean age of $21.6( \pm 2.2)$ years participated in the study. All were healthy and right-handed with no prior history of neurological or psychiatric disorders, learning disabilities, drug abuse, or chronic illness. Participants were asked to refrain from drinking caffeine or alcohol during the 12 hours prior to the recording sessions, and to arrive with clean, dry hair. All participants had an intellectual coefficient (IQ) equal to or greater than 80 , as measured by the Shipley-2 intelligence scale (Shipley, Gruber, Martin, \& Klein, 2009), and showed normal-toabove-normal parameters of attention and memory, as measured by the Digit Detection and Visual Detection subtests, and successive series of the NEUROPSI test (Ostrosky et al., 2012). Informed consent was obtained from all participants, according to the guidelines of the Institutional Ethics Committee. Finally, all procedures involved in the experiment were approved by this Committee and performed in accordance with the ethical standards laid down in the 1964 Helsinki Declaration and its later amendments, or comparable ethical standards.

\section{Mathematical problems}

A total of 40 logical-mathematical word problems were displayed consecutively on a 32 -in. computer screen placed at a distance of 1 meter from participants. The problems in sentence form, as well as the answer (in numeric values), were simultaneously presented on the screen. Taking into account that the answer was always present below the written problem, once the participants solved the problem, they could decide if that answer was correct or incorrect by pressing one of two answer keys on a computer keyboard: " $\mathrm{B}$ " if they were correct, " $\mathrm{M}$ " if not. The time for each problem (simple or complex) finished when the participant press the answer key. Two types of logical-mathematical problems were used: 20 classified as "simple" and 20 as "complex." Text processing was required in both cases. The simple problems consisted of eight words, while the complex problems averaged 25 words. The former consisted of one-digit quantities and required only one operation to obtain the answer. The problem formula was $x=a$ $+b$. The complex problems, in contrast, consisted of two-digit quantities and required three or more operations. The problem formula was $x=a+(a-b)+[\{a+(a-b)\}+c]$ (see Table 1). Clearly, the text-processing demands of the problems that required three or more operations were greater than those of the one-operation problems. To eliminate the possibility that participants might find it easier to solve simple problems after analyzing more challenging ones, all test sessions began with the simple problems, followed by the complex ones. The

Table 1 Examples of the logical-mathematical word problems used in the study

Category Example

Simple There are 3 birds in a tree; 7 more arrive. How many birds are there now?

Jonas has 5 apples and Mary has 4 . How many apples do they have in total?

Hanna has 9 dollars but spent 4 . How many dollars does she have left?

Complex John scored 45 points for his team; 10 more than Joseph. Marie scored 13 more points than John and Joseph together. How many points did they score in total?

Group A has 24 students; 13 less than group B. Group C has 12 more students than groups A and B combined. What is the total number of students?

A store sold 21 sodas in the morning, and 13 more than in the afternoon. At night it sold 10 more than in the morning and afternoon together. How many sodas were sold in total? 
following parameters were measured while participants tried to solve both types of mathematical word problems: number of correct answers and response times.

The response time was considered because the written problem appears on the screen until the participant presses the corresponding answer key. This included the time each participant took to read, reason, and verify the response, to finally press the answer key corresponding to "correct" or "incorrect."

\section{EEG recording and procedure}

Electrode placement followed the international 10-20 system (Jasper, 1958). Due to the specific interests of this study, the recording sites were F3 and F4, considered prefrontal areas; $\mathrm{T} 3$ and T4, considered temporal areas; and P3 and P4, considered parietal areas (Herwig, Satrapi, \& Schönfeldt-Lecuona, 2003; Homan, 1978; Okamoto et al., 2004). EEGs were recorded continuously with eyes open in two conditions: while solving (1) simple and (2) complex problems. In each condition, participants were awake in a sitting position with their heads supported by the head rest of a comfortable chair. All derivations were referred to linked ears with the ground electrode placed on the forehead. EEGs were recorded using a NEXUS 32 device at 24 bits resolution and filters set at 1$50 \mathrm{~Hz}$. Impedance for the EEG electrodes was maintained below $10 \mathrm{kOhms}$. BioTrace $+{ }^{\circledR}$ software was used to sample $(512 \mathrm{~Hz})$ and store the EEG data for off-line processing.

Epoch rejection was performed by both visual and computer-assisted assessment. Signals were examined offline to identify saturated epochs or those that showed noise due to muscle activity, eye movement, or heartbeat. Those epochs were removed by means of a computer program (CHECASEN; Guevara et al. 2010). An off-line digital filter was applied for the frequencies below $1 \mathrm{~Hz}$ and above $50 \mathrm{~Hz}$ using another computer program (FILDIG; Guevara et al. 2005). All artifact-free EEG segments were analyzed using the EEG bands program (Guevara et al. 2014), which used fast Fourier transform to calculate absolute power (AP) and electroencephalographic correlations (rEEG) by applying the Pearson's correlation coefficient between intrahemispheric (F3-T3, F3-P3, T3-P3, F4-T4, F4-P4, T4-P4) and interhemispheric electrodes (F3-F4, T3-T4, P3-P4) for six frequency bands: delta $(\delta, 1.5-3.5 \mathrm{~Hz})$, theta $(\theta, 3.5-7.5 \mathrm{~Hz})$, alpha1 $(\alpha 1,7.5-10.5 \mathrm{~Hz})$, alpha2 $(\alpha 2,10.5-13.5 \mathrm{~Hz})$, beta1 $(\beta 1,13.5-19.5 \mathrm{~Hz})$, beta2 $(\beta 2,19.5-30 \mathrm{~Hz})$, and gamma $(\gamma$, 31-50 Hz).

\section{Statistical analyses}

Behavioral analysis The number of correct answers and the sum of all response times from each participant were obtained. These parameters were then compared between the two types of problem using a Student's $t$ test for correlated groups, considering a significance level equal to, or below, a $p$ value of .05 for all comparisons.

EEG analysis For each type of mathematical problem, sixty 2-s EEG segments were selected and analyzed for each participant - that is, sixty 2-s EEG segments representatives of the simple problems and sixty 2-s EEG segments representatives of the complex problems. Specifically, the start of the EEG recording coincided with the start of the sequence of the 20 simple problems, and after that, with the start of the sequence of the 20 complex problems. Only for the EEG analysis, these segments were aligned at the beginning of the reading of each mathematical problem. Thus, the EEGs were recorded continuously while participants read the problem and perform the mental calculations required to solve it. This alignment at the begin of reading was used for all 20 simple and 20 complex problems to ensure that we would obtain EEG segments representative of the reading, comprehension, reasoning, and solving phases of the logical-mathematical problems. Selection of EEG segments was performed manually during the posterior analysis of the signals, striving to include EEG segments that were representative of all problems and of all phases.

Before conducting the statistical analyses, and to approximate a normal distribution, the AP values were transformed into logarithms and the correlation data into Fisher $Z$ values. EEG AP values (in log) and $Z$ correlation scores for each EEG band were compared between conditions using the Student's $t$ test for correlated groups. The effect sizes $(g)$ of the statistical tests were calculated following Cohen (1988), and the level of significance was set at $p<.05$ for all comparisons.

\section{Results}

\section{Behavioral results}

During performance of the simple logical-mathematical word problems, participants achieved a higher number of correct answers and had a lower mean response time than while solving the complex logical-mathematical word problems (see Table 2).

\section{Absolute power}

While participants were solving the complex problems, a higher AP of the theta $(t=-4.685, p=.00021$, effect size $[d]=1.085)$ and alpha1 $(t=-2.346, p=.03135, d=$ $0.549)$ bands at F3, as well as of the alpha1 $(t=-2.721$, $p=.01454, d=0.6411)$ and alpha2 $(t=-2.137, p=$ $.04739, d=0.506)$ bands at the P3 derivation were observed, compared with performance on the simple problems. Lower APs were obtained for the fast frequencies, betal $(t=2.694, p=.01537, d=0.637)$ and gamma band 
Table 2 Mean \pm standard deviation $(S D)$ of the number of correct answers and response times (in seconds) obtained during performance of the simple and complex logical-mathematical word problem

\begin{tabular}{lllllllll}
\hline & \multicolumn{2}{l}{ Simple problems } & & \multicolumn{2}{l}{ Complex problems } & $t$ & $p$ \\
\cline { 2 - 3 } & Mean & $S D$ & & Mean & \multicolumn{2}{c}{$S D$} & & \\
\hline Correct answers & $19.17^{*}$ & 0.86 & 11.72 & 3.27 & 9.35 & $<.01$ \\
Response times & $192.08^{*}$ & 51.40 & $1,013.62$ & 324.50 & -10.61 & $<.01$ \\
\hline
\end{tabular}

$* p<.05$, as compared with complex mathematical problems

( $t=2.273, p=.03627, d=0.535)$ only in the right temporal area (T4) while participants solved the complex problems compared with the simple ones (see Fig. 1).

\section{Interhemispheric EEG correlation}

During performance of the complex problems, a higher interfrontal (F3-F4) correlation in the alphal $(t=-4.001, p$ $=.00093, d=0.960)$, alpha2 $(t=-2.53, p=.02159, d=$ $0.589)$, and gamma bands $(t=-2.195, p=.04235, d=$ 0.510 ) was obtained compared with the simple problem condition. A similar increase of the correlation was obtained between the T3-T4 derivations in the alphal band $(t=$ $-2.928, p=.00939, d=0.691)$, whereas between the P3-P4 derivations, a higher correlation of the alphal $(t=-3.071, p=$ $.00692, d=0.718)$ and gamma $(t=-2.137, p=.04743, d=$ 0.492 ) bands was observed while participants solved the complex problems compared with the simple ones (see Fig. 2).

\section{Intrahemispheric EEG correlation}

With respect to the left intrahemispheric correlation, a higher value was observed between the F3-P3 derivations in the fast frequencies, betal $(t=-2.373, p=.02973, d=$ $0.557)$, beta2 $(t=-2.342, p=.03159, d=0.557)$, and gamma $(t=-2.435, p=.02620, d=0.569)]$, compared with simple problems (see Fig. 3). In contrast to our observations of the left hemisphere, in the right hemisphere a lower correlation in the delta $(t=2.395, p=.02839, d=$ $0.561)$, theta $(t=2.377, p=.02945, d=0.571)$, alpha1 $(t$ $=2.213, p=.04087, d=0.525)$, and beta1 $(t=2.442, p=$ $.02581, d=0.576)$ bands was obtained between the T4P4 derivations during performance of the complex problems compared with the simple ones (see Fig. 3).

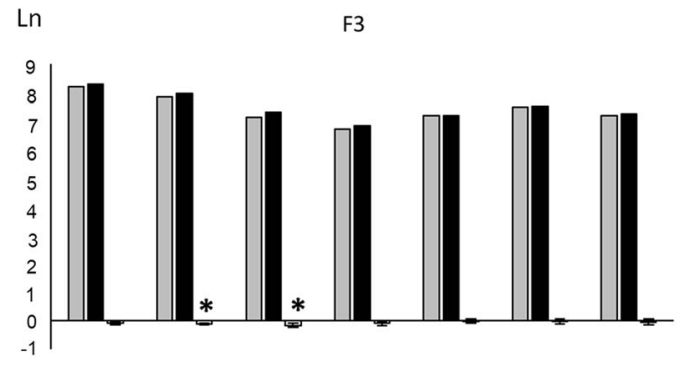

T3

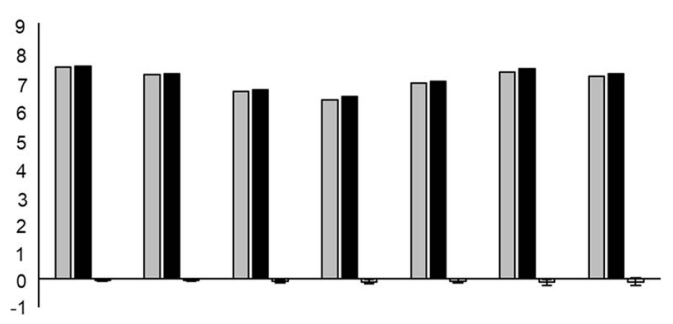

P3

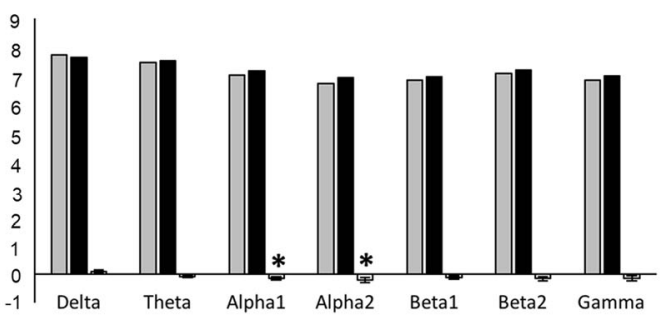

Fig. 1 Mean and mean differences (MD) $\pm 1 S E$ of the absolute power (in natural logarithms; Ln) for each frequency band recorded in the left (F3, T3, and P3) and right (F4, T4, and P4) cortices of the young men while

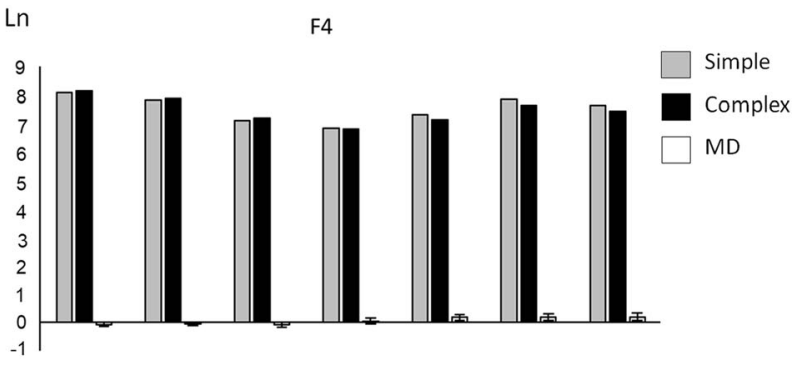

T4

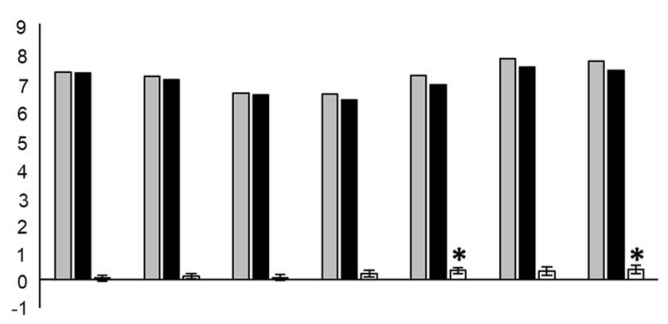

P4

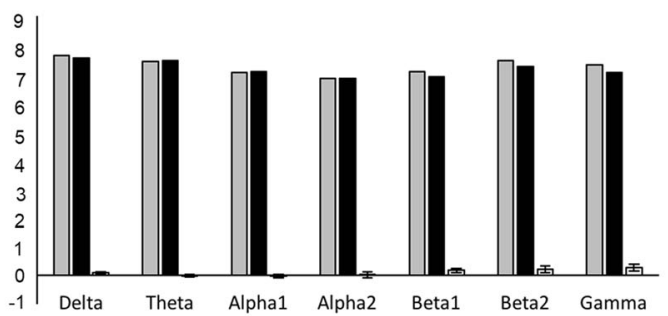

solving simple and complex logical-mathematical word problems. ${ }^{*} p<$ .05 , as compared with simple problems. Note. $S E=$ standard error 

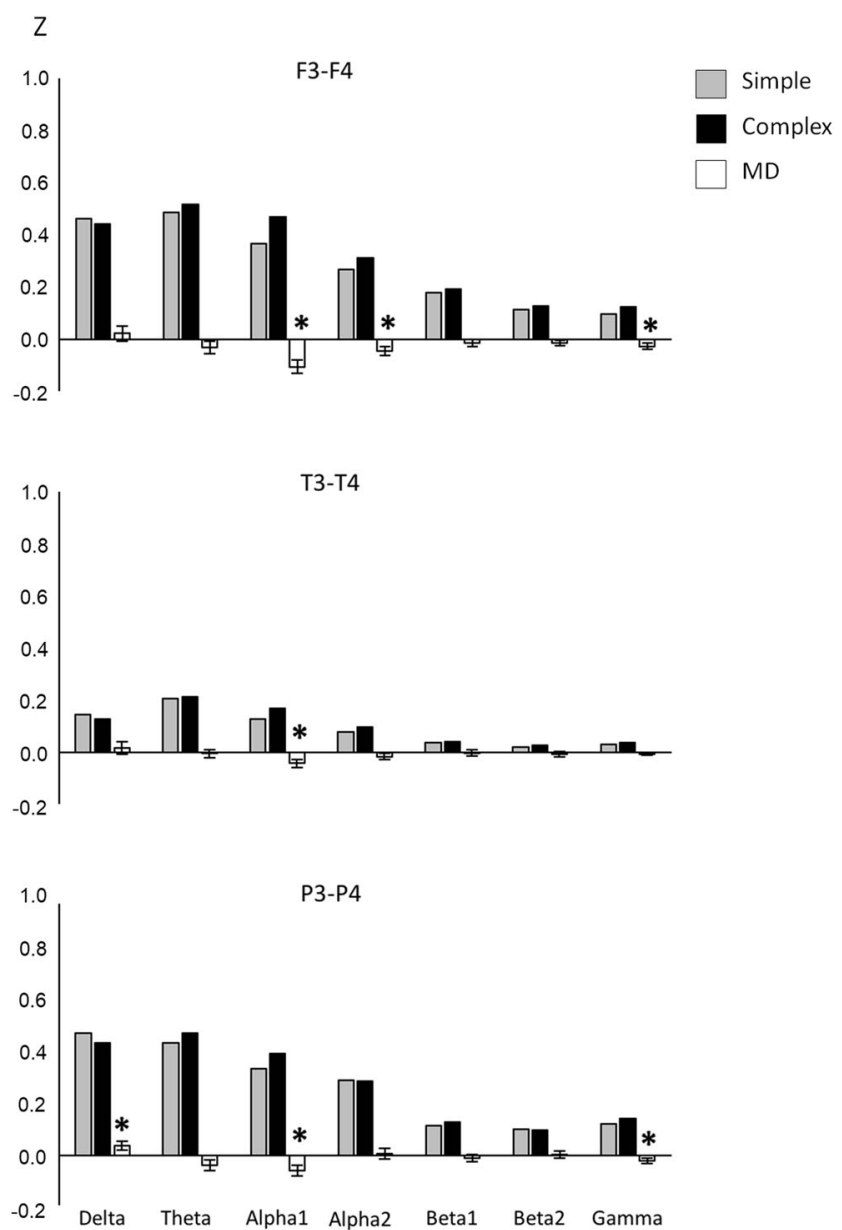

Fig. 2 Mean and mean differences (MD) $\pm 1 S E$ of the interhemispheric correlation (in $z$ values) among the prefrontal (F3-F4), temporal (T3-T4), and parietal (P3-P4) cortices for the different frequency bands recorded in young men while solving simple and complex logical-mathematical word problems. ${ }^{*} p<.05$, as compared with simple problems. Note. $S E=$ standard error

\section{Discussion}

The present study compared the cortical activation and degree of electroencephalographic synchronization between different cortical areas while participants were solving simple versus complex logical-mathematical word problems. The EEG pattern associated with the solving of the simple problems was different from the one observed during the solving of complex problems, which featured a higher AP of the theta and alpha bands in the left frontal and parietal cortices. These results agree with other reports (Dimitriadis et al., 2016; Prabhakaran et al., 2001; Zago et al., 2001) that have shown a higher activation of frontal and parietal areas associated with the increased difficulty of problems. Indeed, our behavioral data showed that participants had fewer correct answers and longer response times when solving the complex problems. These findings reflect the greater complexity of those problems.
Prevalence of the alpha and theta bands has been linked to the maintenance and manipulation of information (Kawasaki, Kitajo, \& Yamaguchi, 2010). In effect, studies have shown that theta is related to the coding phase of short-term memory tasks (Klimesch, Doppelmayr, Russegger, \& Pachinger, 1996), with both information maintenance (Sarnthein, Petsche, Rappelsberger, Shaw, \& von Stein, 1998) and retrieval (Klimesch et al., 2001), and with differences in memory load (Sauseng et al., 2005). Changes in the alpha range, in contrast, have been associated with attentional processes, inhibition of irrelevant information (Cooper et al., 2003; Foxe \& Snyder, 2011; Payne et al., 2013; Sauseng et al., 2005) and mental calculation (Halgren et al., 2002; Palva et al., 2005). Thus, considering that the prefrontal and parietal cortices are involved in mathematical reasoning and calculation processes (Prabhakaran et al., 2001), it is not surprising that these two cortical areas show greater activation of the theta and alpha bands during the solving of complex problems.

Contrary to the activation of the left frontal and parietal regions during the solving of complex problems, a lower activation (indicated by the lower AP of the fast frequencies) was obtained in the right temporal areas. Decreases in temporal regions have been evidenced in other studies; for example, using positron emission tomography, Dehaene et al. (1999) reported a decreased blood flow in the temporal regions during multiplication retrieval compared with a rest condition. Similarly, Zago et al. (2001) reported that during processes of mental calculation that required several intermediate steps to reach a solution (compute condition), temporal deactivation was larger than during the solving of mathematical problems that required only a memory retrieval strategy. Thus, it is probable that — as Zago et al. (2001) suggest — the mental calculations required to solve the complex mathematical problems presented in our study induced a temporal inhibition that was related to cognitive demand and the degree of difficulty of the calculation task. Previous studies that analyzed the comprehension and reasoning of textual material have shown prominent activations in temporal and frontal regions (Fasotti et al., 1992; Fasotti et al., 1994; Grafman et al., 1982; Haier \& Camilla, 1995; Jackson \& Warrington, 1986; Just et al., 1996; Nichelli et al., 1995; Partiot et al., 1996). The fact that the text-processing demands of the problems presented in our study requiring three or more operations were greater than those of the one-operation problems suggests that decreased activation of the temporal areas could be associated with both the higher cognitive demands required to understand the sentences and the greater complexity of the mental calculations required to solve the complex problems even more convincing.

Taken together, these data show that the solving of complex logical-mathematical word problems requires simultaneous activation of the prefrontal and parietal cortices, accompanied by lower activation of temporal areas. This EEG 

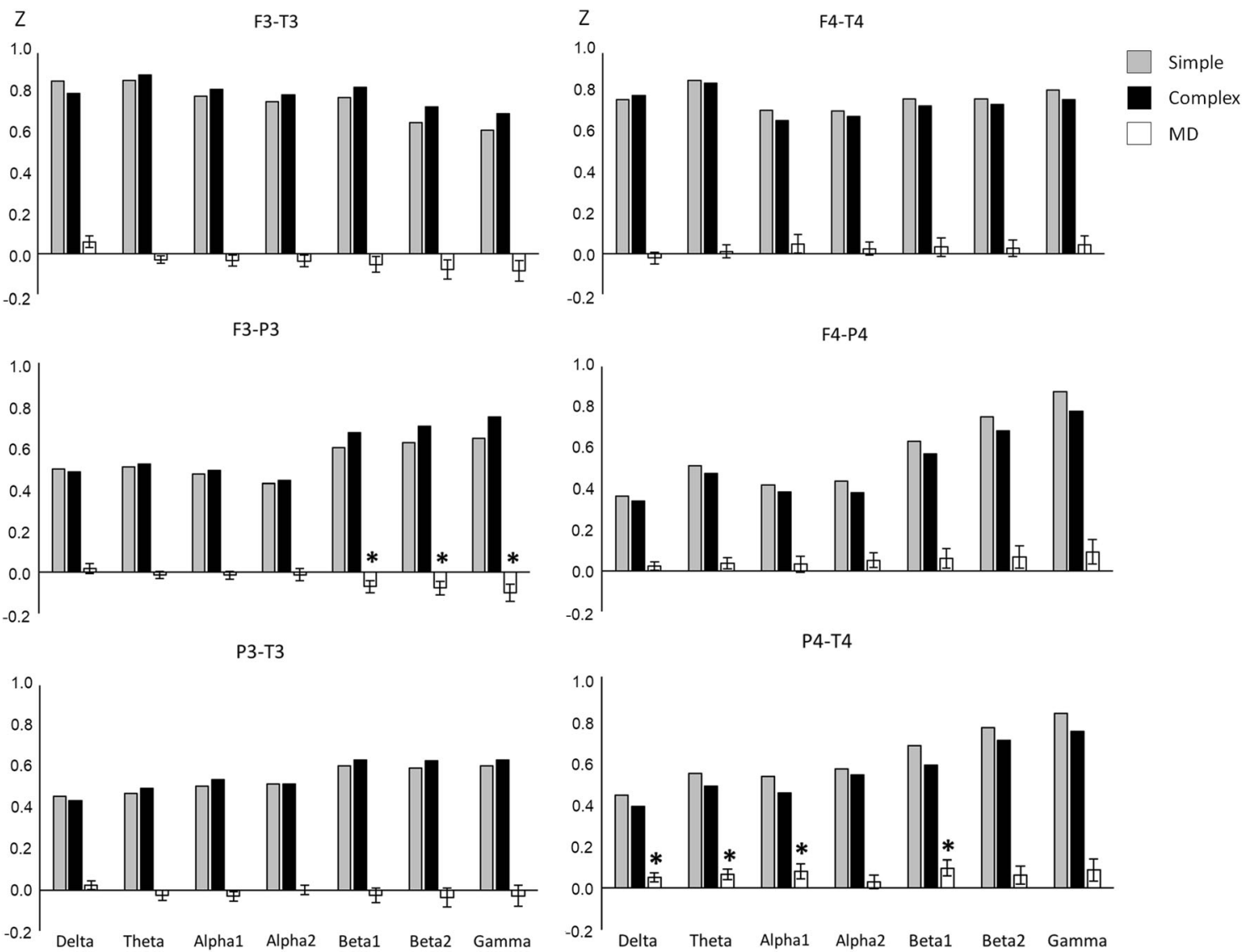

Fig. 3 Mean and mean differences $(\mathrm{MD}) \pm 1 S E$ of the intrahemispheric correlation (in $z$ values) between the left (F3-T3, F3-P3, and P3-T3) and right (F4-T4, F4-P4, and $\mathrm{P} 4-\mathrm{T} 4)$ derivations for the different frequency

bands recorded in young men while solving simple and complex logicalmathematical word problems. ${ }^{*} p<.05$, as compared with simple problems. Note. $S E=$ standard error

pattern could represent a characteristic cortical functioning that involves, on the one hand, activation of a left prefrontoparietal network associated with mathematical reasoning and calculation and, on the other, deactivation of the right temporal region associated with the greater complexity of the text processing and mental calculation.

An additional question of interest is the meaning of the left and right activations observed in this study. Insights concerning the asymmetric roles of the frontal, parietal, and temporal lobes in mathematical reasoning can be drawn from other research, which has reported a pronounced lateralization during mathematical tasks. In fact, several studies on number processing and calculation have concluded that numerical deficits are observed more frequently after left lesions than after right ones (Cipolotti, Butterworth, \& Denes, 1991; Lemer, Dehaene, Spelke, \& Cohen, 2003). Similarly, there are reports that arithmetical tasks usually lead to a strong left dominance (Delazer et al., 2003; Zago et al., 2001), though increased activity in the right areas has also been reported (StanescuCosson et al., 2000). Thus, the higher AP found in our study in the left prefrontal and parietal areas concurs with the research mentioned above and supports the notion that reasoning and mental calculation involve greater participation by cortical areas located in the left hemisphere.

In our study, the solving of the complex problems was also associated with higher interprefrontal (F3-F4) and interparietal (P3-P4) correlations, specifically in the alpha and gamma bands, and with a higher intertemporal correlation (T3-T4) in alpha1. The alpha band has been associated with attentional processes, while gamma has been related to high executive demands (Başar, Başar-Eroglu, Karakaş, \& Schürmann, 2001), retention processes (Sarnthein et al., 1998), and information transfer between regions (Engel \& Fries, 2010). Also, a higher phase synchrony in the alpha band has been reported in occipital, parietal, and frontal regions during periods of mental calculation and working memory (Halgren et al., 2002). Our data agree with those results because, in effect, a higher functional synchronization between hemispheres was observed, specifically in the alpha and gamma range, as our participants solved the complex problems. Hence, we can suggest that a higher degree of alpha and gamma synchronization between hemispheres could be a requisite for maintaining longer sustained attention, manipulating the larger amount of information 
(Anokhin, Lutzenberger, \& Birbaumer, 1999; Jap et al., 2010) and performing the reasoning and mental calculations necessary to solve complex problems.

In addition to the higher correlation between cortical regions of both hemispheres, participants also showed a higher correlation of the fast frequencies (beta1, beta2, gamma) between prefrontoparietal regions of the left hemisphere while solving the complex problems. The fast bands (beta1, beta2, gamma) have been related to interneuronal communication of inhibitory networks (Whittington et al., 2000) and high executive demands (Ahmed \& Cash, 2013; Bașar et al., 2001; Fries, Reynolds, Rorie, Desimone, \& Reynolds, 2001; Haig, Gordon, Wright, Meares, \& Bahramali, 2000; Paul et al., 2005). Indeed, gamma phase synchrony has been proposed as an index of integrative network processing (Phillips \& Singer, 1997). Thus, the higher synchronization of the fast bands between left prefrontoparietal regions could be related to a greater active maintenance and transfer of information between the two cortices, which could be required to perform the higher number of mental calculations involved in solving complex problems.

Unlike the higher correlation observed in the left hemisphere, in the right hemisphere a lower intrahemispheric correlation, mainly of the slow bands (delta, theta, alpha) and betal was seen between right parietal and temporal regions while participants solved the complex problems compared with the simple ones.

The temporal region, as mentioned above, is strongly involved in language processing, and a clear left hemispheric asymmetry has been reported in relation to both sentence listening and reading (Geschwind \& Levitsky, 1986; Toga \& Thompson, 2003). The left parietal cortex also plays a key role in mental arithmetic and reasoning (Jackson \& Warrington, 1986); thus, the lower synchronization of the different EEG bands between right temporal and parietal areas confirms the dominance of the left hemisphere in the reasoning and mental calculation processes that predominated as participants solved the complex logical-mathematical word problems. Support for such hemispheric asymmetry comes from other studies (Deglin \& Kinsbourne, 1996; Nathan, Kintsch, \& Young, 1992; Wharton \& Grafman, 1998), which have proposed that the right hemisphere participates in content-dependent reasoning (using real-world knowledge that is often spatial in nature), whereas the left hemisphere participates in the mathematical formalization of the problem (which is often abstract, symbolic, and nonspatial; Greeno, 1989; Kintsch \& Greeno, 1985). Word problems require making inferences concerning content-dependent reasoning in relation to the mathematical formalization of the problem in order to perform successful mathematical reasoning. The results of our study show that a lower correlation between right parietal and temporal areas occurs during the solving of complex mathematical problems, which could reflect the lesser content-dependent reasoning that participants use to make greater cognitive resources available to focus directly on the mathematical reasoning of the problem.

An important point that must be considered here is that the EEG segments analyzed included segments that were representative of the word-reading, text comprehension, reasoning, and mental calculation phases of both types of problems. Although it is impossible to identify specific EEG changes for each phase, these data support the idea that the functioning of the prefrontal, parietal and temporal cortices in each hemisphere varies according to the difficulty of the task and the degree of cognitive demands (specific mental processes) required to solve each type of mathematical word problem.

In summary, the data from the present study show that solving complex problems require a characteristic pattern of activation and functional synchronization between cortices: (1) an increased left activation (frontal and parietal) associated to a deactivation of the right temporal cortex, (2) an increased rINTER between the three cortices, (3) an, increased rINTRA between left prefrontalparietal cortices, and (4) a functional decoupling of the right parietal-temporal cortices.

The increased intertemporal correlation in the alphal band and the decreased right parietal-temporal correlation in almost all the EEG bands could be explained considering the pivotal role that the temporal cortex plays in the text processing. In fact, as was mentioned, it is probably that an increased correlation between cortices of both hemispheres allows for more sustained attention, manipulating the larger amount of information (Anokhin et al., 1999; Jap et al., 2010) and performing the text reasoning and mental calculations necessary to solve complex problems. On the other hand, taking into account that word problems require making inferences concerning contentdependent reasoning, it is likely that the lower right parietal-temporal correlation was associated with the text processing so that lower content-dependent reasoning was used for the participants to focus directly on the mathematical reasoning of the problem.

In conclusion, the data from the present study show that complex problems require activation of a left prefrontalparietal circuit, probably to maintain and manipulate multiple pieces of information, together with a functional decoupling of the right parietal-temporal cortices to suppress contentdependent reasoning and focus more cognitive resources on the mathematical reasoning required to solve the problem.

Finally, our study has some limitations, including the degree of difficulty and considerable variation in the length of the texts that described the simple and complex logicalmathematical problems. This was revealed by the significant differences in both the number of correct answers and response times between the simple and complex problems (see Table 2). One approach to correct this could be to analyze only an equivalent unit of time from the different trials (e.g., the 
first few seconds of each problem). However, the risk of analyzing the initial seconds in these different problem types is that it might isolate only the neural substrates involved in textprocessing instead of the substrates involved in mathematical reasoning. A second issue is that, due to technical limitations, we were unable to record and analyze the specific EEG activity of the different phases involved in solving the mathematical word problems presented (i.e., word reading, text comprehension, reasoning, and mental calculation). Third, our participants were undergraduate students, so it remains to be determined to what degree our findings can be extrapolated to other populations. These issues may be resolved in future EEG studies.

\section{Compliance with ethical standards}

\section{Conflict of interest None.}

Publisher's note Springer Nature remains neutral with regard to jurisdictional claims in published maps and institutional affiliations.

\section{References}

Ahmed, O. J., \& Cash, S. S. (2013). Finding synchrony in the desynchronized EEG: The history and interpretation of gamma rhythms. Frontiers in Integrative Neuroscience, 7, 58. https://doi. org/10.3389/fnint.2013.00058

Anokhin, A. P., Lutzenberger, W., \& Birbaumer, N. (1999). Spatiotemporal organization of brain dynamics and intelligence: An EEG study in adolescents. International Journal of Psychophysiology, 33(3), 259-273.

Ashcraft, M. H., \& Krause, J. A. (2007). Working memory, math performance, and math anxiety. Psychonomic Bulletin \& Review, 14(2), 243-248. https://doi.org/10.3758/BF03194059

Başar, E., Başar-Eroglu, C., Karakaș, S., \& Schürmann, M. (2001). Gamma, alpha, delta, and theta oscillations govern cognitive processes. International Journal of Psychophysiology, 39(2), 241-248. https://doi.org/10.1016/S0167-8760(00)00145-8

Besnard, J., Allain, P., Aubin, G., Chauviré, V., Etcharry-Bouyx, F., \& Le Gall, D. (2014). An integrative view of Luria's perspective on arithmetic problem solving: the two sides of environmental dependency. Journal of Clinical and Experimental Neuropsychology, 36(1), 88109. https://doi.org/10.1080/13803395.2013.870135

Burbaud, P., Degreze, P., Lafon, P., Franconi, J., Bouligand, B., Bioulac, B., . . . Allard, M. (1995). Lateralization of prefrontal activation during internal mental calculation: A functional magnetic resonance imaging study. Journal of Neurophysiology, 74, 2194-2200.

Carrillo-de la Peña, M. T., \& García-Larrea, L. (2007). Right frontal event related EEG coherence (ERCoh) differentiates good from bad performers of the Wisconsin Card Sorting Test (WCST). Clinical Neurophysiology, 37, 63-75.

Cipolotti, L., Butterworth, B., \& Denes, G. A. (1991). Specific deficit for numbers in a case of dense acalculia. Brain, 114, 2619-2637.

Cohen, J. (1988). Statistical power analysis for the behavioral sciences (2nd ed.). Mahwah, NJ: Erlbaum.

Cooper, N. R., Croft, R. J., Dominey, S. J., Burgess, A. P., \& Gruzelier, J. H. (2003). Paradox lost? Exploring the role of alpha oscillations during externally vs. internally directed attention and the implications for idling and inhibition hypotheses. International Journal of
Psychophysiology, 47(1), 65-74. https://doi.org/10.1016/S01678760(02)00107-1

Cunillera, T., Fuentemilla, L., Periañez, J., Marco-Pallarés, J., Krämer, U. M., Càmara, E., . . Rodríguez-Fornells, A. (2012). Brain oscillatory activity associated with task switching and feedback processing. Cognitive Affective and Behavioral Neuroscience, 12, 16-33.

Deglin, V. L., \& Kinsbourne, M. (1996). Divergent thinking styles of the hemispheres: How syllogisms are solved during transitory hemisphere suppression. Brain and Cognition, 31, 285-307.

Dehaene, S., \& Cohen, L. (1995). Towards an anatomical and functional model of number processing. Mahwah, NJ: Erlbaum.

Dehaene, S., Molko, N., Cohen, L., \& Wilson, A. J. (2004). Arithmetic and the brain. Current Opinion in Neurobiology, 14(2), 218-224. https://doi.org/10.1016/j.conb.2004.03.008

Dehaene, S., Spelke, E., Pinel, P., Stanescu, R., \& Tsivkin, S. (1999). Sources of mathematical thinking: Behavioral and brain-imaging evidence. Science, 284, 970-974.

Delazer, M., Domahs, F., Bartha, L., Brenneis, C., Lochy, A., Trieb, T., \& Benke, T. (2003). Learning complex arithmetic - An fMRI study. Cognitive Brain Research, 18, 76-88.

DeStefano, D., \& LeFevre, J. (2004). The role of working memory in mental arithmetic. European Journal of Cognitive Psychology, 16(3), 353-386. https://doi.org/10.1080/09541440244000328

Dimitriadis, S. I., Sun, Y., Thakor, N. V., \& Bezerianos, A. (2016). Causal interactions between Frontal $\theta$ - Parieto-Occipital $\alpha 2$ predict performance on a mental arithmetic task. Frontiers in Human Neuroscience, 10, 1-17. https://doi.org/10.3389/fnhum.2016.00454

Eger, E., Sterzer, P., Russ, M. O., Giraud, A. L., \& Kleinschmidt, A. (2003). A supramodal number representation in human intraparietal cortex. Neuron, 37(4), 719-725. https://doi.org/10.1016/S08966273(03)00036-9

Engel, A. K., \& Fries, P. (2010). Beta-band oscillations-Signalling the status quo?. Current Opinion in Neurobiology, 20, 156-165.

Fasotti, L., Eling, P. A. T. M., \& Bremer, J. J. C. B. (1992). The internal representation of arithmetic word problem sentences. Brain and Cognition, 20, 245-263.

Fasotti, L., Eling, P. A. T. M., \& Houtem, J. V. (1994). Categorization of arithmetic word problems by normal, frontal and posterior-injured patients. Journal of Clinical and Experimental Neuropsychology, $16,723-733$.

Fias, W. (2001). Two routes for the processing of verbal numbers: Evidence from the SNARC effect. Psychological Research, 65(4), 250-259. https://doi.org/10.1007/s004260100065

Foxe, J. J., \& Snyder, A. C. (2011). The role of alpha-band brain oscillations as a sensory suppression mechanism during selective attention. Frontiers in Psychology, 2, 1-13. https://doi.org/10.3389/fpsyg. 2011.00154

Fries, P., Reynolds, J., Rorie, A., Desimone, R., \& Reynolds, J. (2001). Modulation of oscillatory neuronal synchronization by selective visual attention. Science, 291(5508), 1560-1563. https://doi.org/10. 1126/science. 1055465

Gerig, G., Gouttard, S., \& Corouge, I. (2004). Analysis of brain white matter via fiber tract modeling. Conference Proceedings: Annual International Conference of the IEEE Engineering in Medicine and Biology Society. IEEE Engineering in Medicine and Biology Society, 6, 4421-4424. https://doi.org/10.1109/IEMBS.2004. 1404229

Geschwind, N., \& Levitsky, W. (1986). Left-right asymmetry in temporal speech region. Science, 161, 186-187.

Goel, V., \& Dolan, R. J. (2003). Reciprocal neural response within lateral and ventral medial prefrontal cortex during hot and cold reasoning. NeuroImage, 20(4), 2314-2321. https://doi.org/10.1016/j. neuroimage.2003.07.027

Grafman, J., Passafiume, D., Faglioni, P., \& Boiler, F. (1982). Calculation disturbances in adults with focal hemispheric damage. Cortex, 18, $37-50$. 
Greeno, J. G. (1989). Situation models, mental models, and generative knowledge. In D. K. K. Kotovsky (Ed.), Complex information processing: The impact of Herbert A. Simon (pp. 35-55). Hillsdale, NJ: Erlbaum.

Guevara, M. A., \& Corsi-Cabrera, M. (1996). EEG coherence or EEG correlation? International Journal of Psychophysiology, 23, 145153. https://doi.org/10.1016/S0167-8760(96)00038-4.

Guevara, M.A., Ramos, J., Hernández-González, M., \& Corsi-Cabrera, M. (2005). FILDIG: a program to filter brain electrical signals in the frequency domain. Computer Methods \& Programs in Biomedicine, 80, 93-186.

Guevara, M. A., Sanz-Martin, A., Corsi-Cabrera, M., Amezcua, C., \& Hernández-González, M. (2010). CHECASEN: programa para revisar señales EEG fuera de línea. Revista Mexicana de Ingeniería Biomédica, 31(2), 135-141.

Guevara, M. A., Sanz-Martin, A., \& Hernández-González, M. (2014). EEGbands: A computer program to statistically analyze parameters of electroencephalographic signals. Journal of Behavioral and Brain Science, 4, 308-324.

Haier, R. J., \& Camilla, P. B. (1995). Sex differences and lateralization in temporal lobe glucose metabolism during mathematical reasoning. Developmental Neuropsychology, 11, 405-414.

Haig, A. R., Gordon, E., Wright, J. J., Meares, R. A., \& Bahramali, H. (2000). Synchronous cortical gamma-band activity in task-relevant cognition. NeuroReport, 11(4), 669-675. https://doi.org/10.1097/ 00001756-200003200-00004

Halgren, E., Boujon, C., Clarke, J., Wang, C., \& Chauvel, P. (2002). Rapid distributed fronto-parieto-occipital processing stages during working memory in humans. Cerebral Cortex, 12(7), 710-728. https://doi.org/10.1093/cercor/12.7.710

Harris, A. Z., \& Gordon, J. A. (2015). Long-range neural synchrony in behavior. Annual Review of Neuroscience, 38(1), 171-194. https:// doi.org/10.1146/annurev-neuro-071714-034111

Herwig, U., Satrapi, P., \& Schönfeldt-Lecuona, C. (2003). Using the international 10-20 EEG system for positioning of transcranial magnetic stimulation. Brain Topography, 16(2), 95-99.

Homan, R. W. (1978). Cerebral location of international 10-20 system electrode placement. Electroencephalography and Clinical Neurophysiology, 66, 376-382.

Jackson, M., \& Warrington, E. K. (1986). Arithmetic skills in patients with unilateral cerebral lesions. Cortex, 22, 611-620.

Jap, B. T., Lal, S., \& Fischer, P. (2010). Inter-hemispheric electroencephalography coherence analysis: Assessing brain activity during monotonous driving. International Journal of Psychophysiology, 76(3), 169-173. https://doi.org/10.1016/j.ijpsycho.2010.03.007.

Jasper, H. H. (1958). Report of the committee on methods of clinical examination in electroencephalography. Electroencephalography and Clinical Neurophysiology, 10(2), 370-375. https://doi.org/10. 1016/0013-4694(58)90053-1

Just, M. A., Carpenter, P. A., Keller, T. A., Eddy, W. P., \& Thulborn, K. R. (1996). Brain activation modulated by sentence comprehension. Science, 274, 114-116.

Kawasaki, M., Kitajo, K., \& Yamaguchi, Y. (2010). Dynamic links between theta executive functions and alpha storage buffers in auditory and visual working memory. European Journal of Neuroscience, 31(9), 1683-1689. https://doi.org/10.1111/j.1460-9568.2010.07217

Kintsch, W., \& Greeno, J. G. (1985). Understanding and solving word arithmetic problems. Psychological Review, 92, 109-129.

Klimesch, W., Doppelmayr, M., Russegger, H., \& Pachinger, T. (1996). Theta band power in the human EEG and the encoding of new information. NeuroReport, 7, 1235-1240. https://doi.org/10.1097/ 00001756-199605170-00002

Klimesch, W., Doppelmayr, M., Stadler, W., Pöllhuber, D., Sauseng, P., \& Roehm, D. (2001). Episodic retrieval is reflected by a process specific increase in human electroencephalographic theta activity.
Neuroscience Letters, 302(1), 49-52. https://doi.org/10.1016/ S0304-3940(01)01656-1

Knops, A., \& Willmes, K. (2014). Numerical ordering and symbolic arithmetic share frontal and parietal circuits in the right hemisphere. NeuroImage, 84, 786-795. https://doi.org/10.1016/j.neuroimage. 2013.09.037

Le Clec'H, G., Dehaene, S., Cohen, L., Mehler, J., Dupoux, E., Poline, J. B., \& Le Bihan, D. (2000). Distinct cortical areas for names of numbers and body parts independent of language and input modality. NeuroImage,12(4), 381-391. https://doi.org/10.1006/nimg. 2000.0627

Lemer, C., Dehaene, S., Spelke, E., \& Cohen, L. (2003). Approximated quantities and exact number words: Dissociable systems. Neuropsychologia, 41, 1942-1958.

Leron, U. (2003). Origins of mathematical thinking: A synthesis. European Research in Mathematics Education III. Retrieved from http://www.dm.unipi.it/ didattica/CERME3/proceedings/Groups/ TG1/TG1_leron_cerme3.pdf

Leron, U. (2004). Mathematical thinking and human nature: Consonance and conflict. $P M E, 28(3), 217-224$.

Levin, H. S., Scheller, J., Rickard, T., Grafman, J., Martinkowski, K., Winslow, M., \& Mirvis, S. (1996). Dyscalculia and dyslexia after right hemisphere injury in infancy. Archives of Neurology, 53, 8896.

Mahmood, A., Othman, M. F., \& Yusof, Y. M. (2012). A conceptual framework for mathematical ability analysis through the lens of cultural neuroscience. Procedia - Social and Behavioral Sciences, 56, 175-182. https://doi.org/10.1016/j.sbspro.2012.09.644

Mesulam, M. M. (1994). Neurocognitive networks and selectively distributed processing. Revue Neurologique (Paris), 150, 564-569.

Mori, S., Kaufmann, W. E., Davatzikos, C., Stieltjes, B., Amodei, L., Fredericksen, K., \& Van Zijl, P. C. M. (2002). Imaging cortical association tracts in the human brain using diffusion-tensor-based axonal tracking. Magnetic Resonance in Medicine, 47(2), 215-223. https://doi.org/10.1002/mrm.10074

Nathan, M. J., Kintsch, W., \& Young, E. (1992). A theory of algebraword-problem comprehension and its implications for the design of learning environments. Cognition and Instruction, 9, 329-389.

Nichelli, P., Grafman, J., Pietrini, P., Clark, K., Lee, K. Y., \& Miletich, R. (1995). Where the brain appreciates the moral of a story. Cognitive Neuroscience and Neuropsychology, 6, 2309-2313.

Okamoto, M., Dan, H., Sakamoto, K., Takeo, K., Shimizu, K., Kohno, S., \& Dan, I. (2004). Three-dimensional probabilistic anatomical cranio-cerebral correlation via the international 10-20 system oriented for transcranial functional brain mapping. NeuroImage, 21, 99-111. https://doi.org/10.1016/j.neuroimage.2003.08.026

Ostrosky, F., Gómez, E., Matute, E., Rosselli, M., Ardila, A., \& Pineda, D. (2012). NEUROPSI: Atención y memoria [NEUROPSI: Memory and attention]. Mexico City, Mexico: Manual Moderno.

Palva, J. M., Palva, S., \& Kaila, K. (2005). Phase synchrony among neuronal oscillations in the human cortex. The Journal of Neuroscience, 25, 3962-3972. https://doi.org/10.1523/ JNEUROSCI.4250-04.2005

Partiot, A., Grafman, J., Sadato, N., Flitman, S., \& Wild, K. (1996). Brain activation during script event processing. NeuroReport, 7, 761-766.

Paul, R. H., Richard, C., Lawrence, J., Goldberg, E., Williams, L. M., Cooper, N., . . . Gordon, E. (2005). Age-dependent change in executive function and gamma $40 \mathrm{~Hz}$ phase synchrony. Journal of Integrative Neuroscience, 4(01), 63-76. https://doi.org/10.1142/ S0219635205000690

Payne, L., Guillory, S., \& Sekuler, R. (2013). Attention-modulated alphaband oscillations protect against intrusion of irrelevant information. Journal of Cognitive Neuroscience, 25(9), 1463-1476. https://doi. org/10.1162/jocn a 00395 
Phillips, W. A., \& Singer, W. (1997). In search of common foundations for cortical computation. Behavioral and Brain Sciences, 20(4), 657-683. https://doi.org/10.1017/S0140525X9700160X

Piazza, M., Mechelli, A., Butterworth, B., \& Price, C. J. (2002). Are subitizing and counting implemented as separate or functionally overlapping processes. NeuroImage, 15, 435-446. https://doi.org/ 10.1006/nimg.2001.0980

Prabhakaran, V., Rypma, B., \& Gabrieli, J. D. (2001). Neural substrates of mathematical reasoning: A functional magnetic resonance imaging study of neocortical activation during performance of the necessary arithmetic operations test. Neuropsychology, 15(1), 115-127. https://doi.org/10.1037/0894-4105.15.1.115

Rousell, M., Catherwood, D., Edgar, G., \& Design, A. (2012). An EEG case study of arithmetical reasoning by four individuals varying in imagery and mathematical ability: Implications for mathematics education. Word Academy of Science, Engineering and Technology, 71, 1946-1948.

Sarnthein, J., Petsche, H., Rappelsberger, P., Shaw, G. L., \& Von Stein, A. (1998). Synchronization between prefrontal and posterior association cortex during human working memory. Proceedings of the National Academy of Sciences, 95(12), 7092-7096.https://doi.org/ 10.1073/pnas.95.12.7092

Sauseng, P., Klimesch, W., Doppelmayr, M., Hanslmayr, S., Schabus, M., \& Gruber, W. R. (2004). Theta coupling in the human electroencephalogram during a working memory task. Neuroscience Letters, 354(2), 123-126. https://doi.org/10.1016/j.neulet.2003.10.002

Sauseng, P., Klimesch, W., Schabus, M., \& Doppelmayr, M. (2005). Fronto-parietal EEG coherence in theta and upper alpha reflect central executive functions of working memory. International Journal of Psychophysiology, 57(2), 97-103. https://doi.org/10.1016/j. ijpsycho.2005.03.018

Shaw, C. (1984). Correlation and coherence analysis a selective tutorial review of the EEG. International Journal of Psychophysiology, 1(3), 255-266. https://doi.org/10.1016/0167-8760(84)90045-X

Shaw, J. C., O'Connor, K. P., \& Ongley, C. (1977). The EEG as a measure of cerebral functional organization. British Journal of Psychiatry, 130(3), 260-264.

Shipley, W. C., Gruber, C. P., Martin, T. A., \& Klein, A. M. (2009). Shipley-2: Escala breve de inteligencia [Shipley-2: Brief intelligence scale]. Mexico City, Mexico: Manual Moderno.

Stanescu-Cosson, R., Pinel, P., van de Moortele, P. F., Le Bihan, D., Cohen, L., \& Dehaene, S. (2000). Understanding dissociations in dyscalculia: A brain imaging study of the impact of number size on the cerebral networks for exact and approximate calculation. Brain, $123,2240-2255$.
Subhani, A. R., Malik, A. S., Kamil, N., \& Saad, M. N. M. (2016). Difference in brain dynamics during arithmetic task performed in stress and control conditions. IECBES 2016: IEEE-EMBS Conference on Biomedical Engineering and Sciences, 1, 695-698. https://doi.org/10.1109/IECBES.2016.7843539

Thatcher, R. W., Biver, C. J., \& North, D. (2007). Spatial-temporal current source correlations and cortical connectivity. Clinical EEG and Neuroscience: Official Journal of the EEG and Clinical Neuroscience Society (ENCS),38(1), 35-48. https://doi.org/10. 1177/155005940703800109

Toga, A. W., \& Thompson, P. M. (2003). Mapping brain asymmetry. Nature Reviews Neuroscience, 4, 37-48.

Varela, F., Lachaux, J., Rodriguez, E., \& Martinerie, J. (2001). The brain web: Phase synchronization and large-scale integration. Nature Reviews, 2, 229-239.

Waisman, I., Leikin, M., Shaul, S., \& Leikin, R. (2014). Brain activity associated with translation between graphical and symbolic representations of functions in generally gifted and excelling in mathematics adolescents. International Journal of Science and Mathematics Education, 12, 669-696. https://doi.org/10.1007/ s10763-014-9513-5

Wang, X., Chen, Z., Zhao, L., \& Zou, S. (2010). The study of mental arithmetic load by EEG data. International Conference on Multimedia Technology, 2010, 1-4. https://doi.org/10.1109/ ICMULT.2010.5629620

Wharton, C., \& Grafman, J. (1998). Deductive reasoning and the brain. Trends in Cognitive Sciences, 2, 54-59.

Whittington, M. A., Traub, R. D., Kopell, N., Ermentrout, B., \& Buhl, E. H. (2000). Inhibition-based rhythms: Experimental and mathematical observations on network dynamics. International Journal of Psychophysiology, 38(3), 315-336. https://doi.org/10.1016/S01678760(00)00173-2

Zago, L., Pesenti, M., Mellet, E., Crivello, F., Mazoyer, B., \& TzourioMazoyer, N. (2001). Neural correlates of simple and complex mental calculation. NeuroImage, 13(2), 314-27. https://doi.org/10.1006/ nimg.2000.0697

Zarjam, P., Epps, J., Lovell, N. H., \& Fang Chen, F. (2012). Characterization of memory load in an arithmetic task using nonlinear analysis of EEG signals. Annual International Conference of the IEEE Engineering in Medicine and Biology Society, 2012, 3519-3522. https://doi.org/10.1109/EMBC.2012.6346725 